\title{
Images - Solitary fibrous tumor of the prostate
}

Anamika Mishra ${ }^{1}$; Mark T. Corkum, MD, MSc ${ }^{1}$; Stephen E. Pautler, MD, FRCSC ${ }^{2,3}$; Bret Wehrli, MD, FRCPC ${ }^{4}$; Eric Winquist, MD, MSc, FRCPC, FACP ${ }^{5}$

${ }^{1}$ Division of Radiation Oncology, Department of Oncology, Western University, London, ON, Canada; ${ }^{2}$ Division of Urology, Department of Surgery, Western University, London, ON, Canada; ${ }^{3}$ Division of Surgical Oncology, Department of Oncology, Western University, London, ON, Canada; ${ }^{4}$ Department of Pathology and Laboratory Medicine, Western University, London, ON, Canada; ${ }^{5}$ Division of Medical Oncology, Department of Oncology, Western University, London, ON, Canada

Cite as: Can Urol Assoc J 2020 June 5; Epub ahead of print. http://dx.doi.org/10.5489/cuaj.6289 Published online June 5, 2020

$* * *$

\section{Introduction}

Solitary fibrous tumour (SFT) is a spindle cell neoplasm of mesenchymal origin. Although first described arising in a subpleural location, ${ }^{1}$ with increased recognition of its histological features, SFTs have been reported to arise at many extrapleural anatomical sites. ${ }^{2}$ SFT of the prostate is extremely rare with fewer than 25 cases reported in the literature to date. ${ }^{3-5}$ Due to the rarity of prostatic SFT, the variation of clinical and morphological appearances of SFT, and the large number of different soft tissue tumour types, prostatic SFT remain difficult to diagnose. ${ }^{6}$ The majority of case reports of prostatic SFTs have short-term follow-up and have been reported in men between the ages of 50 and $70 . .^{3,7}$

Herein we report the case of a 28 -year-old male presenting with urinary obstructive symptoms resulting in the diagnosis of a prostatic SFT. He was treated with a simple suprapubic prostatectomy and has no evidence of local recurrence 14 years later.

\section{Case report}

A 28-year-old male presented with a 2-year history of decreasing urinary flow and suprapubic pressure. He voided every hour and strained to pass urine. There was no hematuria, history of urinary tract infection or nephrolithiasis. He had lost 60 pounds in the past year. There was no significant past medical history. Rectal exam revealed a very large, firm prostate without nodules or induration. Cystoscopy and a CT of the abdomen and pelvis showed bladder trabeculation and significant outflow obstruction secondary to prostatic hypertrophy with a very large median lobe, with an estimated prostate volume of $120 \mathrm{~cm}^{3}$. 
A limited transurethral resection of the prostate performed for diagnostic purposes revealed a cytologically bland, moderately cellular spindle cell neoplasm with the lesional cells arranged in a haphazard or "patternless pattern" and set in a background fibrous stroma containing thick, wiry collagen bundles and numerous blood vessels that often had a branching, staghorn-like pattern (Figure 1). Mitoses were rare (less than 1 per $10 \mathrm{HPF}$ ) and necrosis was not identified. Imunohistochemically, the spindle lesional cells stained strongly and diffusely positively for CD34 and failed to stain for PSA, S100, cytokeratin 8/18, and smooth muscle actin. Staging investigations found no evidence of metastatic disease. MRI of the pelvis revealed a $5.8 \times 6.4 \times 6.5 \mathrm{~cm}$ solid enhancing tumour associated with the superior aspect of the prostate, bulging into the lumen of the urinary bladder (Figure 2).

Local excision was agreed to be the most suitable course of action. After discussion with the patient regarding radical versus simple surgical approaches, a simple suprapubic prostatectomy with suprapubic cystostomy was performed. During the procedure, the mass was excised along with a portion of the bladder neck. The surgical margins were negative. STAT6 immunohistochemical staining, performed retrospectively as this antibody was not available in our lab until 2015, was positive with characteristic nuclear staining of the lesional cells. Following the procedure, the patient was able to void normally.

Long-term clinical follow-up, 14 years after his surgery, reveals no evidence of recurrence, with a $\mathrm{CT}$ scan of the abdomen and pelvis 12 years after his surgery demonstrating no radiographic evidence of local recurrence. Some urinary frequency and urgency were noted, and a rectal exam revealed a firm, enlarged prostate with a thick perineum. Cystoscopic examination was normal.

\section{Discussion}

First described in 1931 by Klemperer and Rabin, SFT have been found to occur most frequently in the 6th and 7 th decades of life, with about $80 \%$ of cases behaving in a benign manner. ${ }^{8,9}$ SFTs can be difficult to diagnose, characterize biologically, and treat given their wide range of clinical and morphological features. ${ }^{4}$ Immunophenotypically, expression of CD34, Bc12, and CD99 is characteristic but not specific whereas STAT6 immunopositivity is quite specific given the presence of a NAB2-STAT6 gene fusion on chromosome 12q13 in the great majority of SFTs. ${ }^{1,4}$

Moureau-Zabotto reported the largest series of prostatic SFT, comprising 21 cases. $^{7}$ All patients except one presented with non-specific or obstructive urinary symptoms. All patients had normal serum PSA levels. ${ }^{7}$ Of the 21 patients, 17 had follow-up that ranged from 2 months to 10 years. Of these 17,1 patient had a local recurrence and 2 patients died of post-operative complications. In general, complete resection of a SFT has been found to be an important predictive factor for positive clinical outcomes. ${ }^{8}$

Our case is unique for both the young age of the patient as well as its long-term follow-up without evidence of recurrence. The only case of a SFT in a younger patient was reported in 
2002 by Grasso et al. who described a prostatic SFT that was removed via a radical prostatectomy using a bladder neck sparing technique. ${ }^{10}$ However, no follow-up was provided.

\section{Conclusions}

Treatment options for SFT of the prostate include a transurethral resection of the prostate, simple prostatectomy, radical prostatectomy, pelvic exenteration and pelvic tumor resection. ${ }^{3}$ Although most solitary fibrous tumours are benign, the behaviour of SFT can be unpredictable with $10 \%$ behaving aggressively with local recurrence or distant metastasis despite the lack of conventional histological features of malignancy. As such, complete tumour resection with negative margins is advisable, with chemotherapy or radiotherapy reserved for non-resectable or metastatic tumours. ${ }^{3,5,7}$ In our case, long-term control was achieved through a simple prostatectomy rather than a radical approach; however, we believe that treatment must be individualized. 


\section{References}

1. Demicco EG, Meyer C. Solitary fibrous tumor. In: Shah S, Collins KA, eds. Uptodate. Vol 41; 2019:28-32. [updated 2019 March 22; cited 2019 Sept 20]. https://www.uptodate.com/contents/solitary-fibrous-tumor. Accessed September 20, 2019.

2. Ronchi A, Cozzolino I, Zito Marino F, et al. Extrapleural solitary fibrous tumor: A distinct entity from pleural solitary fibrous tumor. An update on clinical, molecular and diagnostic features. Ann Diagn Pathol 2018;34:142-150.

3. Yang W, Sun F, Liu H, et al. Solitary fibrous tumors of the prostate: A case report. Oncol Lett 2015;10(3):1617-1619.

4. Ronchi A, La Mantia E, Gigantino V, et al. A rare case of malignant solitary fibrous tumor in prostate with review of the literature. Diagn Pathol 2017;12(1):4-9.

5. Tanaka Y, Nakamoto A, Inada Y, et al. A case of malignant solitary fibrous tumor of the prostatic urethra. BJR|case reports 2018;4(4):20180034.

6. Mentzel T, Bainbridge TC, Katenkamp D. Solitary fibrous tumour: Clinicopathological, immunohistochemical, and ultrastructural analysis of 12 cases arising in soft tissues, nasal cavity and nasopharynx, urinary bladder and prostate. Virchows Arch 1997;430(6):445-453.

7. Moureau-Zabotto L, Chetaille B, Bladou F, et al. Solitary fibrous tumor of the prostate: Case report and review of the literature. Case Rep Oncol 2012;5(1):22-29.

8. Robinson LA. Solitary fibrous tumors of the pleura. Curr Opin Pulm Med 2012;18(4):339-346.

9. Klemperer P, Rabin C. Primary neoplasms of the pleura. A report of five cases. Arch Pathol 1931;11:385-412.

10. Grasso M, Blanco S, Franzoso F, et al. Solitary fibrous tumor of the prostate. Acta Urol Jpn. 2004;50(6):405-407. 


\section{Figures and Tables}

Fig. 1. Solitary fibrous tumour histology demonstrating a moderately cellular, cytologically bland spindle cell neoplasm with the lesional cells arranged in a haphazard or "patternless pattern." It is set in a background fibrous stroma containing thick, wiry collagen bundles, and numerous blood vessels that often had a branching, staghorn-like pattern.

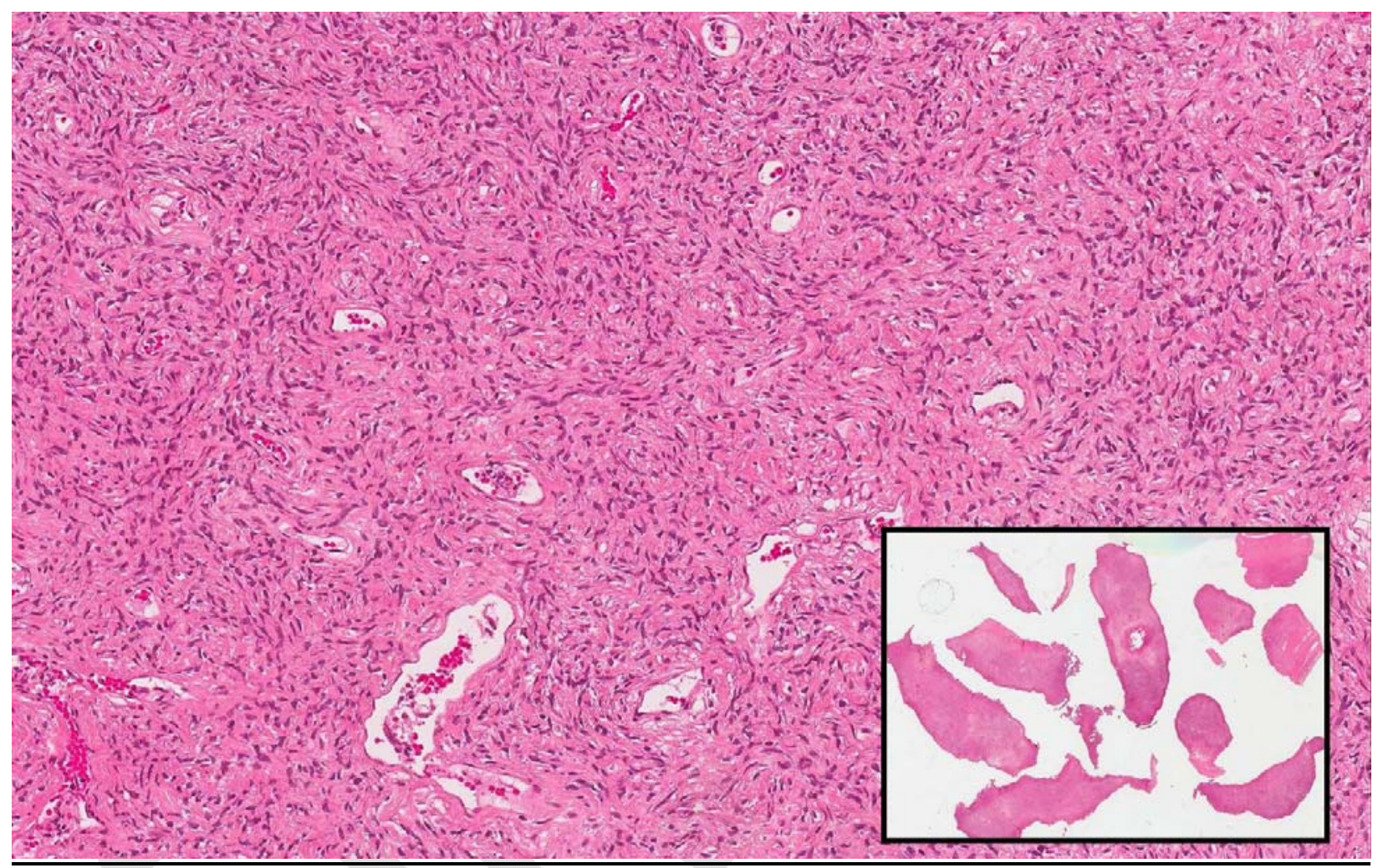


Fig. 2. Sagittal T1 post gadolinium magnetic resonance imaging scan demonstrating the solitary fibrous tumor of the prostate (red arrow). Note the Foley catheter (green arrow) inflated in the prostatic urethra, which was subsequently advanced into the bladder.

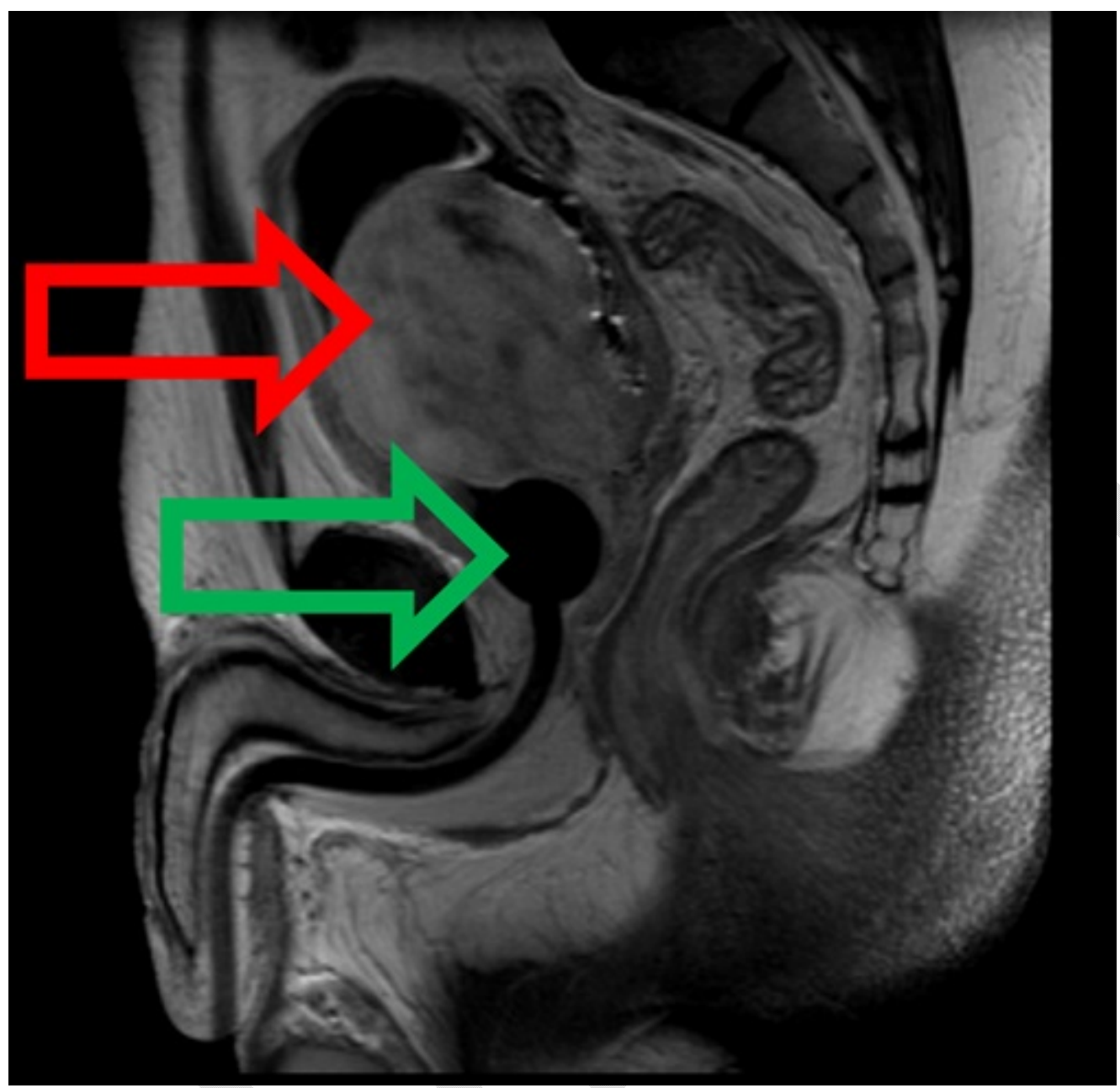

